\title{
Effect of Antifoaming Agent on Colorectal Benign Tumours in Colonoscopy: A Meta-Analysis
}

\author{
Hu Zhang \\ central hospital of wuhan \\ Jing Liu \\ central hopital of wuhan \\ Lin Song Ma \\ central hospital of wuhan
}

Heng Zhang ( $\sim$ 497697662@qq.com)

\section{Research article}

Keywords: Antifoaming agent, Simethicone, Polyethylene glycol, Colonoscopy, Meta-analysis, Systematic review

Posted Date: June 24th, 2020

DOI: https://doi.org/10.21203/rs.3.rs-30593/v1

License: (c) (7) This work is licensed under a Creative Commons Attribution 4.0 International License. Read Full License 


\section{Abstract}

Background: Although several trials have showed that addition of antifoaming agent to polyethylene glycol (PEG) can improve bowel preparation, whether PEG plus antifoaming agent has a beneficial role in detection of benign tumours during colonoscopy has yet to be confirmed. Our aim was to clarify whether adding simethicone to PEG solution could improve the effect of detection of colorectal benign tumours.

Methods: The PubMed, EMBASE, and Cochrane Library databases were searched for articles published prior to September 2019. The outcomes included the detection rates of colorectal adenomas and polyps.

Results: Twenty studies were eligible. Although there was no difference in the colorectal adenoma detection rate (ADR), a significant effect of simethicone for diminutive adenomas $(<10 \mathrm{~mm}$ ) was revealed in the group with simethicone. We also found that simethicone could significantly improve the ADR in proximal colon, but did not affect the colorectal polyp detection rate (PDR). Furthermore, the subgroup analyses revealed that the beneficial effect of simethicone in the ADR among Asian $(P=0.005)$ and $A D R<25 \%(P=0.003)$. Moreover, it was a significant finding that the low dose simethicone was as effective as the high dose one with respect to the detection of colorectal benign tumours.

Conclusions: In summary, the addition of simethicone to PEG might benefit to improve diminutive adenomas in the right colon for colonoscopy in Asia. The low-dose simethicone was recommend to the detection of colorectal benign tumours. However, large clinical trials are necessary to validate our results and determine the ideal dose of simethicone.

\section{Background}

Colorectal cancer (CRC) is one of the most common cancers worldwide. The incidence and mortality of CRC have been rapidly increasing in Asian countries [1, 2]. Early diagnosis is associated with better survival and quality of life. Currently, colonoscopy is a standard first line tool in screening, surveillance, and prevention of colorectal tumours $[3,4]$. The colorectal adenoma detection rate (ADR) has been recognized as the most indicator of the quality of colonoscopy. In the consensus statement, polyethylene glycol (PEG) is recommended as the first choice for bowel preparation [5]. However, up to $24 \%$ of patients have been shown to have a poor quality of bowel preparation [6]. Inadequate bowel preparation is associated with an increased risk of missed colorectal benign tumours and greater discomfort for the patient [7-9].

Simethicone, preventing bubble formation and gas retention to alleviate bloating, is an effective and safe antifoaming agent during endoscopic procedures. A combination of simethicone and PEG has been shown to improve the visualization of the bowel for colonoscopy. Thus, simethicone may have a theoretical benefit in the detection of benign tumours in colonoscopy, especially diminutive lesions.

A large number of previous studies have evaluated the effect of simethicone in ADR during colonoscopy, but the results are inconsistent. Hence, a recently meta-analysis would be necessary. However, whether simethicone plus PEG has a beneficial role in detection of benign tumours during colonoscopy has yet to be confirmed. Therefore, we performed a meta-analysis to investigate the effect of detection of colorectal benign tumours.

\section{Methods}

\section{Literature search}

The literature was searched in PubMed, EMBASE, and Cochrane Central databases (up to September 1, 2019) using the keywords 'colonoscopy', 'antifoaming agent' or 'simethicone', and 'randomized'. We also performed a manual search of the reference list of published articles.

\section{Inclusion criteria}

(1) Study design: randomized studies as a full manuscript;

(2) Language: limited to English;

(3) Population: patients who underwent a colonoscopy;

(4) Controls: PEG without simethicone for bowel preparation;

(5) Intervention: PEG with simethicone for bowel preparation;

(6) Outcomes: 1) primary endpoints: colorectal adenoma and polyp detection rate (ADR and PDR), 2) secondary endpoints: adverse events.

\section{Exclusion criteria}

(1) Bowel preparation without PEG or simethicone;

(2) Non-human studies;

(3) Duplicate publications; 
(4) Studies without available data.

\section{Data Extraction}

The three investigators $(\mathrm{HZ}, \mathrm{JL}$ and $\mathrm{LM})$ were independently assigned to extract the data from each primary manuscript. Discrepancies or disagreements were resolved by consensus. The extracted data included the first author, year of publication, country or region, number of patients, study design, detailed information of interventions and controls (ADR and PDR), and adverse events.

\section{Assessment of Study Quality}

The Cochrane Collaboration's risk of bias tool [10], in which the description of sequence generation, allocation concealment, blinding of participants, personnel and outcome assessors, incomplete outcome data and selective outcome reporting assessed, was used to evaluate the quality of the randomized studies. The quality scale was assessed as 'low risk of bias', 'unclear risk of bias' and 'high risk of bias'.

\section{Data Syntheses and Statistical Analyses}

The odds ratio (OR) was used for discrete variables, and the mean difference (MD) and standardized difference in mean (SDM) were used for continuous variables. The pooled OR and 95\% confidence intervals (Cls) were calculated from each study using either a fixed-effects model (Mantel-Haenszel method) or a random-effects model (Der Simonian and Laird method). When the heterogeneity was significant, the random-effects model was used in the pooled data; otherwise, the fixed-effects model was used. Heterogeneity among the studies was assessed using $\mathrm{I}^{2}$ statistic or $\chi^{2}$ test. $\mathrm{I}^{2}>50 \%$ or $\mathrm{P}<$ 0.10 were considered to represent significant heterogeneity. The publication bias was evaluated by the Egger's test, where $P<0.1$ in a two-tailed test was considered to be positive. In the subgroup analyses, $\mathrm{P}<0.05$ for the $\chi^{2}$ test indicated statistically a significant heterogeneity. For all the pooled results, a sensitivity analysis was conducted to examine the robustness by excluding one or more studies each time [11]. All the statistical analyses and plots were performed using the Review Manager statistical software version 5.0 (The Cochrane Collaboration, Copenhagen, Denmark) and Stata version 12.0 (Stata Corp LLC, Texas, USA).

\section{Results}

\section{Study Selection}

The literature search retrieved 169 citations, 96 of which were excluded because they were duplicates. Of the 73 potentially eligible studies, 53 publications were excluded, and 20 studies focused on comparing PEG with and without simethicone evaluated the effect of ADR and PDR. This metaanalysis was conducted in accordance with the standard guidelines of the Preferred Reporting Items for Systematic Reviews and Meta-Analysis (PRISMA) [12] (Fig. 1).

\section{Study characteristics}

The 20 studies [13-32] included 6306 patients, of whom 3162 and 3144 patients were assigned to the PEG plus simethicone group and PEG group, respectively (Tables 1 and 2). These studies were performed in five countries (China, Korean, Italy, the USA, and the Netherlands). 
Table 1

Characteristics of the studies included in the meta-analysis

\begin{tabular}{|c|c|c|c|c|c|c|c|c|c|c|c|c|}
\hline \multirow{2}{*}{$\begin{array}{l}\text { Author } \\
\text { (year) }\end{array}$} & \multirow{2}{*}{\multicolumn{2}{|c|}{ Groups }} & \multirow[t]{2}{*}{$\mathrm{N}$} & \multirow{2}{*}{$\begin{array}{l}\text { Dose of } \\
\text { simethicone } \\
\text { (mg) }\end{array}$} & \multirow{2}{*}{$\begin{array}{l}\text { Bubble } \\
\text { score }\end{array}$} & \multirow{2}{*}{$\begin{array}{l}\text { Insertion } \\
\text { time (min) }\end{array}$} & \multirow{2}{*}{$\begin{array}{l}\text { Withdraw } \\
\text { time (min) }\end{array}$} & \multicolumn{5}{|c|}{ Adverse events } \\
\hline & & & & & & & & bloating & nausea & vomiting & $\begin{array}{l}\text { abdominal } \\
\text { pain }\end{array}$ & $\begin{array}{l}\text { sleep } \\
\text { disorder }\end{array}$ \\
\hline \multirow{2}{*}{$\begin{array}{l}\text { Rishi } \\
\text { (2019) }\end{array}$} & NS & $2 L$ & 84 & \multirow[t]{2}{*}{200} & $\begin{array}{l}1.77 \pm \\
1.00\end{array}$ & $\begin{array}{l}5.48 \pm \\
2.82\end{array}$ & $\begin{array}{l}11.23 \pm \\
3.99\end{array}$ & NR & 20 & 6 & 12 & NR \\
\hline & S & $\begin{array}{l}2 \mathrm{~L} \\
+ \\
\operatorname{Sim}\end{array}$ & 84 & & $\begin{array}{l}1.20 \pm \\
0.60\end{array}$ & $\begin{array}{l}6.06 \pm \\
3.55\end{array}$ & $\begin{array}{l}11.73 \pm \\
5.52\end{array}$ & NR & 13 & 4 & 10 & NR \\
\hline \multirow{2}{*}{$\begin{array}{l}\text { Morave } \\
\text { (2019) }\end{array}$} & NS & $4 \mathrm{~L}$ & 139 & \multirow[t]{2}{*}{480} & $\begin{array}{l}2.1 \pm \\
2.15\end{array}$ & $\begin{array}{l}6.19 \pm \\
4.62\end{array}$ & $6.65 \pm 1.28$ & NR & NR & NR & NR & NR \\
\hline & S & $\begin{array}{l}4 \mathrm{~L} \\
+ \\
\operatorname{Sim}\end{array}$ & 129 & & $\begin{array}{l}0.1 \pm \\
0.15\end{array}$ & $\begin{array}{l}6.06 \pm \\
3.71\end{array}$ & $6.60 \pm 1.15$ & NR & NR & NR & NR & NR \\
\hline \multirow{2}{*}{$\begin{array}{l}\text { Zhang } \\
(2018)\end{array}$} & NS & $2 \mathrm{~L}$ & 290 & \multirow[t]{2}{*}{1200} & $\begin{array}{l}2.5 \pm \\
0.7\end{array}$ & $7.5 \pm 5.1$ & NR & 59 & 57 & 20 & 24 & 57 \\
\hline & S & $\begin{array}{l}2 \mathrm{~L} \\
+ \\
\operatorname{Sim}\end{array}$ & 289 & & $\begin{array}{l}2.8 \pm \\
0.5\end{array}$ & $6.3 \pm 3.1$ & NR & 34 & 61 & 24 & 21 & 53 \\
\hline \multirow{2}{*}{$\begin{array}{l}\text { Bai } \\
\text { (2018) }\end{array}$} & NS & $2 \mathrm{~L}$ & 286 & \multirow[t]{2}{*}{1200} & $\begin{array}{l}3.98 \pm \\
2.50\end{array}$ & $\begin{array}{l}7.55 \pm \\
4.19\end{array}$ & $6.87 \pm 2.03$ & 57 & 38 & 27 & 9 & NR \\
\hline & S & $\begin{array}{l}2 \mathrm{~L} \\
+ \\
\operatorname{Sim}\end{array}$ & 290 & & $\begin{array}{l}1.00 \pm \\
1.26\end{array}$ & $\begin{array}{l}7.84 \pm \\
5.12\end{array}$ & $6.47 \pm 1.80$ & 23 & 39 & 30 & 11 & NR \\
\hline \multirow{2}{*}{$\begin{array}{l}\text { Yoo } \\
(2016)\end{array}$} & NS & $2 \mathrm{~L}$ & 130 & \multirow[t]{2}{*}{400} & NR & $\begin{array}{l}6.75 \pm \\
5.13\end{array}$ & $\begin{array}{l}17.29 \pm \\
13.17\end{array}$ & 71 & 51 & 15 & 31 & 39 \\
\hline & S & $\begin{array}{l}2 \mathrm{~L} \\
+ \\
\operatorname{Sim}\end{array}$ & 130 & & NR & $\begin{array}{l}6.78 \pm \\
3.78\end{array}$ & $\begin{array}{l}13.35 \pm \\
7.86\end{array}$ & 31 & 54 & 8 & 7 & 36 \\
\hline \multirow{2}{*}{$\begin{array}{l}\text { Zorzi } \\
(2016)\end{array}$} & NS & $2 \mathrm{~L}$ & 924 & \multirow[t]{2}{*}{ NR } & NR & NR & $10.4 \pm 29.9$ & NR & NR & NR & NR & NR \\
\hline & S & $\begin{array}{l}2 \mathrm{~L} \\
+ \\
\operatorname{Sim}\end{array}$ & 940 & & NR & NR & $10.6 \pm 30.0$ & NR & $N R$ & NR & NR & NR \\
\hline \multirow{2}{*}{$\begin{array}{l}\text { Kump } \\
(2018)\end{array}$} & NS & $2 \mathrm{~L}$ & 193 & \multirow[t]{2}{*}{ NR } & NR & NR & NR & 28 & 26 & 3 & 37 & NR \\
\hline & S & $\begin{array}{l}2 \mathrm{~L} \\
+ \\
\text { Sim }\end{array}$ & 194 & & NR & NR & $N R$ & 26 & 26 & 1 & 34 & NR \\
\hline \multirow{2}{*}{$\begin{array}{l}\text { Parente } \\
(2015)\end{array}$} & NS & $4 \mathrm{~L}$ & 189 & \multirow[t]{2}{*}{ NR } & NR & $12 \pm 7$ & $10 \pm 3$ & NR & NR & NR & NR & 43 \\
\hline & $S$ & $\begin{array}{l}2 \mathrm{~L} \\
+ \\
\operatorname{Sim}\end{array}$ & 193 & & NR & $13 \pm 7$ & $11 \pm 6$ & NR & $N R$ & NR & NR & 37 \\
\hline \multirow{2}{*}{$\begin{array}{l}\text { Mussetto } \\
\text { (2014) }\end{array}$} & NS & $4 \mathrm{~L}$ & 60 & \multirow[t]{2}{*}{ NR } & NR & $7.8 \pm 5.1$ & $13.8 \pm 9.6$ & 21 & 20 & NR & 6 & 26 \\
\hline & S & $\begin{array}{l}2 \mathrm{~L} \\
+ \\
\operatorname{Sim}\end{array}$ & 60 & & NR & $6.5 \pm 3.5$ & $11.4 \pm 9.4$ & 15 & 23 & NR & 9 & 17 \\
\hline \multirow{2}{*}{$\begin{array}{l}\text { Leone } \\
\text { (2013) }\end{array}$} & NS & $4 \mathrm{~L}$ & 79 & \multirow[t]{2}{*}{ NR } & NR & $9.8 \pm 3.6$ & NR & 1 & 7 & 2 & 2 & 3 \\
\hline & S & $\begin{array}{l}2 \mathrm{~L} \\
+ \\
\operatorname{Sim}\end{array}$ & 78 & & NR & $10.9 \pm 6.1$ & $N R$ & 1 & 5 & 6 & 5 & 7 \\
\hline \multirow{2}{*}{$\begin{array}{l}\text { Valiante } \\
\text { (2013) }\end{array}$} & NS & $4 \mathrm{~L}$ & 126 & 160 & $N R$ & NR & $N R$ & 33 & 26 & $N R$ & 5 & NR \\
\hline & $S$ & $\begin{array}{l}2 \mathrm{~L} \\
+ \\
\text { Sim }\end{array}$ & 138 & & NR & NR & $N R$ & 11 & 27 & NR & 13 & NR \\
\hline Cesaro & NS & $4 \mathrm{~L}$ & 51 & 160 & NR & $9.5 \pm 5.8$ & $7.0 \pm 1.8$ & 12 & 23 & NR & 2 & NR \\
\hline (2013) & & & & & & & & & & & & \\
\hline $\begin{array}{l}\text { S: PEG wit } \\
\text { Preparatio }\end{array}$ & & one & up; & PEG group o & Sim: sir & icone; $\mathrm{N}$ : & I number o & ients in & ded; NR & treport; E & PS: Boston & \\
\hline
\end{tabular}




\begin{tabular}{|c|c|c|c|c|c|c|c|c|c|c|c|c|}
\hline & $S$ & $\begin{array}{l}2 \mathrm{~L} \\
+ \\
\operatorname{Sim}\end{array}$ & 50 & & NR & $8.1 \pm 3.8$ & $7.6 \pm 2.4$ & 4 & 10 & NR & 6 & NR \\
\hline \multirow{2}{*}{$\begin{array}{l}\text { Gentile } \\
(2013)\end{array}$} & NS & $2 \mathrm{~L}$ & 60 & \multirow[t]{2}{*}{160} & NR & NR & NR & NR & 6 & 3 & 1 & 0 \\
\hline & $S$ & $\begin{array}{l}4 \mathrm{~L} \\
+ \\
\operatorname{Sim}\end{array}$ & 60 & & NR & $N R$ & NR & NR & 12 & 4 & 1 & 0 \\
\hline \multirow{2}{*}{$\begin{array}{l}\text { Matro } \\
(2012)\end{array}$} & NS & $2 \mathrm{~L}$ & 61 & \multirow[t]{2}{*}{400} & NR & NR & NR & 32 & 18 & 3 & 21 & 16 \\
\hline & $S$ & $\begin{array}{l}2 \mathrm{~L} \\
+ \\
\operatorname{Sim}\end{array}$ & 62 & & NR & NR & NR & 25 & 22 & 3 & 17 & 16 \\
\hline \multirow{2}{*}{$\begin{array}{l}\text { Repici } \\
(2012)\end{array}$} & NS & $2 \mathrm{~L}$ & 190 & \multirow[t]{2}{*}{160} & NR & $7.3 \pm 3.5$ & NR & 43 & 57 & NR & 30 & NR \\
\hline & $S$ & $\begin{array}{l}2 \mathrm{~L} \\
\stackrel{+}{\operatorname{Sim}}\end{array}$ & 187 & & NR & $7.9 \pm 3.7$ & NR & 47 & 60 & NR & 34 & NR \\
\hline \multirow{2}{*}{$\begin{array}{l}\text { Jansen } \\
\text { (2011) }\end{array}$} & NS & $2 \mathrm{~L}$ & 102 & \multirow[t]{2}{*}{ NR } & NR & NR & NR & NR & NR & NR & 12 & NR \\
\hline & $S$ & $\begin{array}{l}2 \mathrm{~L} \\
+ \\
\operatorname{Sim}\end{array}$ & 86 & & NR & NR & NR & NR & NR & NR & 9 & NR \\
\hline \multirow{2}{*}{$\begin{array}{l}\text { Pontone } \\
\text { (2011) }\end{array}$} & NS & $2 \mathrm{~L}$ & 72 & \multirow[t]{2}{*}{160} & NR & NR & NR & NR & 7 & 4 & 2 & 1 \\
\hline & $S$ & $\begin{array}{l}4 \mathrm{~L} \\
\stackrel{+}{\operatorname{Sim}}\end{array}$ & 72 & & NR & NR & NR & NR & 16 & 5 & 1 & 1 \\
\hline \multirow{2}{*}{$\begin{array}{l}\text { Lazzaroni } \\
\text { (1993) }\end{array}$} & NS & $4 \mathrm{~L}$ & 48 & \multirow[t]{2}{*}{120} & NR & NR & NR & 26 & 23 & NR & 15 & 21 \\
\hline & $S$ & $\begin{array}{l}4 \mathrm{~L} \\
+ \\
\operatorname{Sim}\end{array}$ & 57 & & NR & NR & NR & 26 & 20 & NR & 13 & 11 \\
\hline \multirow{2}{*}{$\begin{array}{l}\text { McNally } \\
\text { (1989) }\end{array}$} & NS & NR & 12 & \multirow[t]{2}{*}{160} & $\begin{array}{l}0.778 \pm \\
0.278\end{array}$ & NR & NR & NR & NR & NR & NR & NR \\
\hline & $S$ & $N R$ & 14 & & $\begin{array}{l}0.18 \pm \\
0.054\end{array}$ & NR & NR & NR & NR & NR & NR & NR \\
\hline \multirow{2}{*}{$\begin{array}{l}\text { McNally } \\
\text { (1988) }\end{array}$} & NS & NR & 48 & \multirow[t]{2}{*}{80} & $\begin{array}{l}0.696 \pm \\
0.112\end{array}$ & NR & NR & NR & NR & NR & NR & NR \\
\hline & $S$ & NR & 49 & & $\begin{array}{l}0.114 \pm \\
0.05\end{array}$ & NR & NR & NR & NR & NR & NR & NR \\
\hline
\end{tabular}


Table 2

ADR and PDR of the studies included in the meta-analysis

\begin{tabular}{|c|c|c|c|c|c|c|c|c|c|c|c|c|c|c|c|c|}
\hline \multirow{3}{*}{$\begin{array}{l}\text { Author } \\
\text { (year) }\end{array}$} & \multirow[t]{3}{*}{ Country } & \multicolumn{2}{|c|}{ Groups } & \multirow[t]{3}{*}{$\mathrm{N}$} & \multicolumn{6}{|c|}{ Adenoma } & \multicolumn{6}{|c|}{ Polyp } \\
\hline & & & & & $\mathrm{N}$ & $\%$ & $\begin{array}{l}\text { Left } \\
\text { colon }\end{array}$ & $\begin{array}{l}\text { Right } \\
\text { colon }\end{array}$ & $\begin{array}{l}< \\
10\end{array}$ & $\geqq$ & $\mathrm{~N}$ & $\%$ & $\begin{array}{l}\text { Left } \\
\text { colon }\end{array}$ & $\begin{array}{l}\text { Right } \\
\text { colon }\end{array}$ & $\begin{array}{l}< \\
10\end{array}$ & $\geq 10 \mathrm{~mm}$ \\
\hline & & & & & & & & & $\mathrm{mm}$ & & & & & & $\mathrm{mm}$ & \\
\hline \multirow{2}{*}{$\begin{array}{l}\text { Rishi } \\
\text { (2019) }\end{array}$} & \multirow[t]{2}{*}{ USA } & NS & $2 \mathrm{~L}$ & 84 & NR & NR & NR & NR & NR & NR & 46 & 54.8 & NR & NR & NR & NR \\
\hline & & $S$ & $\begin{array}{l}2 \mathrm{~L}+ \\
\mathrm{Sim}\end{array}$ & 84 & NR & NR & NR & NR & NR & NR & 47 & 56.0 & NR & NR & $\mathrm{NR}$ & NR \\
\hline \multirow{2}{*}{$\begin{array}{l}\text { Morave } \\
\text { (2019) }\end{array}$} & \multirow[t]{2}{*}{ USA } & NS & $4 \mathrm{~L}$ & 139 & 54 & 38.8 & NR & NR & NR & NR & 69 & 49.6 & NR & NR & NR & NR \\
\hline & & S & $\begin{array}{l}4 \mathrm{~L}+ \\
\text { Sim }\end{array}$ & 129 & 43 & 33.3 & NR & NR & NR & NR & 60 & 46.5 & NR & NR & NR & NR \\
\hline \multirow{2}{*}{$\begin{array}{l}\text { Zhang } \\
(2018)\end{array}$} & \multirow[t]{2}{*}{ China } & NS & $2 \mathrm{~L}$ & 290 & 45 & 15.5 & 22 & 30 & 46 & 6 & 93 & 32.1 & 64 & 46 & $\mathrm{NR}$ & NR \\
\hline & & $S$ & $\begin{array}{l}2 \mathrm{~L}+ \\
\mathrm{Sim}\end{array}$ & 289 & 64 & 22.1 & 36 & 48 & 78 & 6 & 98 & 33.9 & 67 & 62 & NR & NR \\
\hline \multirow[t]{2}{*}{ Bai } & \multirow[t]{2}{*}{ China } & NS & $2 \mathrm{~L}$ & 286 & 41 & 14.3 & 35 & 32 & 60 & 7 & 85 & 29.7 & NR & NR & NR & NR \\
\hline & & $S$ & $\begin{array}{l}2 \mathrm{~L}+ \\
\mathrm{Sim}\end{array}$ & 290 & 61 & 21.0 & 49 & 85 & 122 & 12 & 109 & 37.6 & NR & NR & NR & $N R$ \\
\hline \multirow{2}{*}{$\begin{array}{l}\text { Yoo } \\
(2016)\end{array}$} & \multirow[t]{2}{*}{ Korea } & NS & $2 \mathrm{~L}$ & 130 & 60 & 46.2 & NR & NR & NR & NR & NR & NR & NR & NR & NR & NR \\
\hline & & $S$ & $\begin{array}{l}2 \mathrm{~L}+ \\
\mathrm{Sim}\end{array}$ & 130 & 65 & 50 & NR & NR & NR & NR & NR & NR & NR & NR & $\mathrm{NR}$ & NR \\
\hline \multirow{2}{*}{$\begin{array}{l}\text { Zorzi } \\
(2016)\end{array}$} & \multirow[t]{2}{*}{ Italy } & NS & $2 \mathrm{~L}$ & 924 & 346 & 37.4 & NR & NR & NR & NR & 569 & 61.6 & NR & NR & 403 & 166 \\
\hline & & $S$ & $\begin{array}{l}2 \mathrm{~L}+ \\
\mathrm{Sim}\end{array}$ & 940 & 322 & 34.3 & NR & NR & NR & NR & 542 & 57.7 & NR & NR & 380 & 162 \\
\hline \multirow{2}{*}{$\begin{array}{l}\text { Kump } \\
(2018)\end{array}$} & \multirow[t]{2}{*}{ Austria } & NS & $2 \mathrm{~L}$ & 193 & NR & NR & NR & NR & NR & NR & NR & NR & NR & NR & NR & NR \\
\hline & & S & $\begin{array}{l}2 \mathrm{~L}+ \\
\mathrm{Sim}\end{array}$ & 194 & NR & NR & NR & NR & NR & NR & NR & NR & NR & NR & NR & NR \\
\hline \multirow{2}{*}{$\begin{array}{l}\text { Parente } \\
\text { (2015) }\end{array}$} & \multirow{2}{*}{ Italy } & NS & $4 \mathrm{~L}$ & 189 & NR & NR & NR & NR & NR & NR & 89 & 49.2 & NR & NR & 61 & NR \\
\hline & & $S$ & $\begin{array}{l}2 \mathrm{~L}+ \\
\mathrm{Sim}\end{array}$ & 193 & NR & NR & NR & NR & NR & NR & 91 & 48.1 & NR & NR & 59 & NR \\
\hline \multirow{2}{*}{$\begin{array}{l}\text { Mussetto } \\
\text { (2014) }\end{array}$} & \multirow{2}{*}{ Italy } & NS & $4 \mathrm{~L}$ & 60 & NR & NR & NR & NR & NR & NR & NR & NR & NR & NR & NR & NR \\
\hline & & $S$ & $\begin{array}{l}2 \mathrm{~L}+ \\
\mathrm{Sim}\end{array}$ & 60 & NR & NR & NR & NR & NR & NR & NR & NR & NR & NR & NR & NR \\
\hline \multirow{2}{*}{$\begin{array}{l}\text { Leone } \\
(2013)\end{array}$} & Italy & NS & $4 \mathrm{~L}$ & 79 & 34 & 44.7 & NR & NR & NR & NR & NR & NR & NR & NR & NR & NR \\
\hline & & $S$ & $\begin{array}{l}2 \mathrm{~L}+ \\
\mathrm{Sim}\end{array}$ & 78 & 34 & 43.6 & NR & NR & NR & NR & NR & NR & NR & NR & NR & NR \\
\hline Valiante & Italy & NS & $4 \mathrm{~L}$ & 126 & NR & NR & NR & NR & NR & NR & 71 & 56.3 & NR & NR & 55 & 16 \\
\hline (2013) & & $S$ & $\begin{array}{l}2 \mathrm{~L}+ \\
\mathrm{Sim}\end{array}$ & 138 & NR & NR & NR & NR & NR & NR & 105 & 76.1 & NR & NR & 84 & 21 \\
\hline Cesaro & Italy & NS & $4 \mathrm{~L}$ & 51 & 17 & 34.7 & NR & NR & NR & NR & NR & NR & NR & NR & NR & NR \\
\hline (2013) & & $S$ & $\begin{array}{l}2 \mathrm{~L}+ \\
\mathrm{Sim}\end{array}$ & 50 & 17 & 32.7 & NR & NR & NR & NR & NR & NR & NR & NR & NR & NR \\
\hline Gentile & Italy & NS & $2 \mathrm{~L}$ & 60 & NR & NR & NR & NR & NR & NR & NR & NR & NR & NR & NR & NR \\
\hline (2013) & & $S$ & $\begin{array}{l}4 \mathrm{~L}+ \\
\mathrm{Sim}\end{array}$ & 60 & NR & NR & NR & NR & NR & NR & NR & NR & NR & NR & NR & NR \\
\hline Matro & USA & NS & $2 \mathrm{~L}$ & 61 & 20 & 32.8 & NR & NR & NR & NR & 29 & 47.5 & NR & NR & $\mathrm{NR}$ & NR \\
\hline (2012) & & $S$ & $\begin{array}{l}2 \mathrm{~L}+ \\
\mathrm{Sim}\end{array}$ & 62 & 15 & 24.2 & NR & NR & NR & NR & 23 & 37.1 & NR & NR & NR & NR \\
\hline
\end{tabular}




\begin{tabular}{|c|c|c|c|c|c|c|c|c|c|c|c|c|c|c|c|c|}
\hline Repici & Italy & NS & $2 \mathrm{~L}$ & 190 & NR & NR & NR & NR & NR & NR & NR & NR & NR & NR & NR & NR \\
\hline (2012) & & S & $\begin{array}{l}2 \mathrm{~L}+ \\
\mathrm{Sim}\end{array}$ & 187 & NR & NR & NR & NR & NR & NR & NR & NR & NR & NR & NR & NR \\
\hline Jansen & Netherlands & NS & $2 \mathrm{~L}$ & 102 & NR & NR & NR & NR & NR & NR & 14 & 13.7 & NR & NR & NR & NR \\
\hline (2011) & & S & $\begin{array}{l}2 \mathrm{~L}+ \\
\mathrm{Sim}\end{array}$ & 86 & NR & NR & NR & NR & NR & NR & 23 & 26.7 & NR & NR & NR & NR \\
\hline Pontone & Italy & NS & $2 \mathrm{~L}$ & 72 & 9 & 12.5 & 8 & 1 & NR & NR & 13 & 18.1 & NR & NR & NR & NR \\
\hline (2011) & & S & $\begin{array}{l}4 \mathrm{~L}+ \\
\mathrm{Sim}\end{array}$ & 72 & 12 & 16.7 & 5 & 7 & NR & NR & 22 & 30.6 & NR & NR & NR & NR \\
\hline Lazzaroni & Italy & NS & $4 \mathrm{~L}$ & 48 & NR & NR & NR & NR & NR & NR & NR & NR & NR & NR & NR & NR \\
\hline (1993) & & $S$ & $\begin{array}{l}4 \mathrm{~L}+ \\
\mathrm{Sim}\end{array}$ & 57 & NR & NR & NR & NR & NR & NR & NR & NR & NR & NR & NR & NR \\
\hline McNally & USA & NS & PEG & 12 & NR & NR & NR & NR & NR & NR & NR & NR & NR & NR & NR & NR \\
\hline (1989) & & $S$ & $\begin{array}{l}\text { PEG } \\
+ \\
\text { Sim }\end{array}$ & 14 & NR & NR & NR & NR & NR & NR & NR & NR & NR & NR & NR & NR \\
\hline McNally & USA & NS & PEG & 48 & NR & NR & NR & NR & NR & NR & NR & NR & NR & NR & NR & NR \\
\hline (1988) & & $S$ & $\begin{array}{l}\text { PEG } \\
\stackrel{+}{\text { Sim }}\end{array}$ & 49 & NR & NR & NR & NR & NR & NR & NR & NR & NR & NR & NR & NR \\
\hline
\end{tabular}

\section{Quality assessment}

The Cochrane Collaboration's risk of bias tool was used to evaluate the quality of the randomized studies. Although all studies were single-blind to the examiner, the participants and the blinding of outcome assessment were not influence. Thereby the risk bias of selective reporting of each trial was considered low risk. The quality assessment of randomized studies is shown in supplementary table.

\section{Outcomes}

\section{Primary endpoints}

\section{Colorectal adenoma detection rate (ADR)}

For the primary endpoint, 9 studies reported data on the ADR, included 4069 patients (2042 patients treated with PEG plus simethicone and 2027 patients treated with PEG). The overall ADR during colonoscopy was similar in both groups: $30.9 \%$ in the PEG group and $31.0 \%$ in the PEG plus simethicone group. The heterogeneity among the studies was not significant $\left(I^{2}=41 \% ; P=0.10\right)$. According to the fixed-effects model, the pooled OR was not significant $(O R=1.01 ; 95 \% \mathrm{Cl}, 0.88-1.15 ; P=0.94)$, which suggested that there was a not statistically significant difference in the ADR during colonoscopy between the two groups (Fig. 2). The Begg's funnel plots and Egger's regression test revealed that there was no significant effect of publication bias on the overall adenoma detection rate $(P=0.307)$

\section{Colorectal polyp detection rate (PDR)}

Overall, the PDR was available in 10 studies, including 4544 patients (2279 patients treated with PEG plus simethicone and 2265 patients treated with PEG). The overall PDR during colonoscopy was higher in the group with simethicone (49.1\% vs $48.0 \%)$. The heterogeneity among the studies was significant $\left(I^{2}=64 \% ; \mathrm{P}=0.003\right)$. The pooled $\mathrm{OR}$, according to a random-effects model for PDR $(\mathrm{OR}=1.13 ; 95 \% \mathrm{Cl}, 0.89-1.42 ; \mathrm{P}=0.31)$, was not statistically significant difference between the two groups (Fig. 3). The Egger's regression test revealed that there was no significant effect of publication bias on the overall PDR $(P=0.221)$.

\section{Secondary endpoints}

\section{Adverse events}

16 studies reported data on adverse events, included bloating, vomiting, nausea, abdominal pain and sleep disturbance. Simethicone significantly reduced the incidence of bloating $(15.8 \%$ vs. $25.3 \%)$. Except for bloating $(\mathrm{OR}=0.52 ; 95 \% \mathrm{Cl}, 0.44-0.63, \mathrm{P}<0.00001)$, there were no statistically significant difference in other adverse events. The Egger's regression test revealed that there was no significant effect of publication bias.

\section{Sensitivity analyses}


We performed further sensitivity analyses to assess the impact on the heterogeneity by the exclusion of one or more studies at a time. There was statistically significant heterogeneity for ADR in the right colon (heterogeneity $P=0.09, I^{2}=58 \%$ ). When Bai's study was excluded, it no longer showed heterogeneity of ADR (heterogeneity $P=0.18, I^{2}=45 \%$ ). The other two outcomes had significant heterogeneity, included PDR and the adverse event of bloating. When Valiante's study was excluded, it no longer showed heterogeneity of PDR. The studies associated with the heterogeneity of each outcome were listed in Table 3. 
Table 3

Sensitivity analyses and subgroup analyses of the studies included in the meta-analysis

\begin{tabular}{|c|c|c|c|c|c|c|}
\hline & $\begin{array}{l}\text { Number of } \\
\text { trials }\end{array}$ & $\begin{array}{l}\text { Number of } \\
\text { patients }\end{array}$ & OR/MD (95\%CL) & P value & $1^{2}$ & Study associated with heterogeneity \\
\hline \multicolumn{7}{|l|}{ Primary outcome } \\
\hline ADR & 9 & 4069 & $1.01(0.88-1.15)$ & 0.94 & $41 \%$ & - \\
\hline \multicolumn{7}{|l|}{ Proportion of ADR } \\
\hline$<25 \%$ & 3 & 1299 & $1.55(1.16-2.07)$ & 0.003 & $0 \%$ & - \\
\hline$\geq 25 \%$ & 6 & 2770 & $0.88(0.76-1.03)$ & 0.12 & $0 \%$ & - \\
\hline \multicolumn{7}{|c|}{ Dose of simethicone } \\
\hline$\geq 400 \mathrm{mg}$ & 5 & 1806 & $1.21(0.97-1.50)$ & 0.09 & $50 \%$ & - \\
\hline$<400 \mathrm{mg}$ and NR & 4 & 2263 & $0.89(0.75-1.06)$ & 0.20 & $0 \%$ & \\
\hline \multicolumn{7}{|l|}{ Size of adenoma } \\
\hline$<10 \mathrm{~mm}$ & 2 & 1155 & $2.36(1.79-3.10)$ & $<.00001$ & $29 \%$ & - \\
\hline$\geq 10 \mathrm{~mm}$ & 2 & 1155 & $1.39(0.67-2.86)$ & 0.38 & $0 \%$ & - \\
\hline \multicolumn{7}{|c|}{ Location of adenoma } \\
\hline Right colon & 3 & 1299 & $2.61(1.43-4.76)$ & 0.002 & $58 \%$ & Bai $2018\left(1^{2}=45 \%\right)$ \\
\hline Left colon & 3 & 1299 & $1.44(1.02-2.02)$ & 0.04 & $23 \%$ & - \\
\hline \multicolumn{7}{|l|}{$\begin{array}{l}\text { Regions of the } \\
\text { populations }\end{array}$} \\
\hline Asia & 3 & 1415 & $1.45(1.12-1.87)$ & 0.005 & $0 \%$ & - \\
\hline Not-Asia & 5 & 2386 & $0.88(0.74-1.04)$ & 0.14 & $0 \%$ & - \\
\hline PDR & 10 & 4544 & $1.13(0.89-1.42)$ & 0.31 & $64 \%$ & Valiante 2013 (I2 = 41\%) \\
\hline \multicolumn{7}{|c|}{ Dose of simethicone } \\
\hline$\geq 400 \mathrm{mg}$ & 4 & 1546 & $1.06(0.80-1.41)$ & 0.67 & $40 \%$ & \\
\hline$<400 \mathrm{mg}$ and NR & 6 & 2998 & $1.23(0.85-1.79)$ & 0.28 & $74 \%$ & Valiante $2013(12=41 \%)$ \\
\hline \multicolumn{7}{|l|}{ Size of adenoma } \\
\hline$<10 \mathrm{~mm}$ & 3 & 2498 & $0.93(0.79-1.09)$ & 0.37 & $46 \%$ & - \\
\hline$\geq 10 \mathrm{~mm}$ & 2 & 2128 & $0.98(0.78-1.22)$ & 0.84 & $0 \%$ & - \\
\hline \multicolumn{7}{|l|}{ Proportion of PDR } \\
\hline$<40 \%$ & 4 & 1487 & $1.29(0.97-1.72)$ & 0.08 & $31 \%$ & - \\
\hline$\geq 40 \%$ & 6 & 3057 & $1.03(0.75-1.41)$ & 0.86 & $67 \%$ & Valiante $2013(12=0 \%)$ \\
\hline \multicolumn{7}{|l|}{$\begin{array}{l}\text { Regions of the } \\
\text { populations }\end{array}$} \\
\hline Asia & 2 & 1155 & $1.24(0.95-1.62)$ & 0.11 & $14 \%$ & - \\
\hline Not-Asia & 8 & 3389 & $1.10(0.82-1.47)$ & 0.53 & $66 \%$ & Valiante $2013\left(I^{2}=22 \%\right)$ \\
\hline \multicolumn{7}{|c|}{ Secondary outcome } \\
\hline \multicolumn{7}{|l|}{ Adverse events } \\
\hline bloating & 11 & 3049 & $0.51(0.36-0.73)$ & 0.0002 & $67 \%$ & Repici $2012\left(I^{2}=49 \%\right)$ \\
\hline nausea & 14 & 3397 & $1.03(0.87-1.22)$ & 0.69 & $33 \%$ & - \\
\hline vomiting & 9 & 2514 & $1.02(0.75-1.40)$ & 0.89 & $0 \%$ & - \\
\hline abdominal pain & 15 & 3669 & $0.89(0.72-1.10)$ & 0.29 & $42 \%$ & - \\
\hline
\end{tabular}

OR, odds ratio; MD, mean difference; Cls, confidence intervals; ADR, detection rate of colorectal adenoma; PDR, detection rate of colorectal polyp. 


\begin{tabular}{|c|c|c|c|c|c|c|}
\hline & $\begin{array}{l}\text { Number of } \\
\text { trials }\end{array}$ & $\begin{array}{l}\text { Number of } \\
\text { patients }\end{array}$ & OR/MD (95\%CL) & $\mathrm{P}$ value & $\mathrm{P}^{2}$ & Study associated with heterogeneity \\
\hline sleep disturbance & 9 & 1990 & $0.81(0.64-1.01)$ & 0.06 & $25 \%$ & - \\
\hline
\end{tabular}

\section{Subgroup analyses}

The results of the subgroup analyses for the ADR and PDR in relation to sites of colorectal adenomas or polyps (right or left colon), sizes of adenomas or polyps ( $\geq 10 \mathrm{~mm}$ or $<10 \mathrm{~mm}$ ), populations (Asia or non-Asia), dose of simethicone ( $\geq 400 \mathrm{mg}$ or $<400 \mathrm{mg}$ and NR), and proportion of $\operatorname{ADR}(\geq 25 \%$ or $<25 \%$ ) are shown in Table 3.

The analysis separately revealed that there was no significant difference $(O R=1.39,95 \% \mathrm{Cl}: 0.67-2.86, P=0.38)$ and heterogeneity $\left(P=0.48, I^{2}=0 \%\right)$ between the two groups for $A D R \geq 10 \mathrm{~mm}$. However, our study displayed a significant increase in the ADR for small adenomas ( $<10 \mathrm{~mm})$ during colonoscopy in the group with simethicone $(\mathrm{OR}=2.36 ; 95 \% \mathrm{Cl}, 1.79-3.10 ; \mathrm{P}<0.00001)$ (Fig. 4a).

When analyzed separately, a significantly higher proportion of ADR in the right colon was present in the PEG plus simethicone group (21.5\% vs $9.7 \%$, OR $=2.61,95 \% \mathrm{Cl} 1.43-4.76, \mathrm{P}=0.002$ ) (Fig. $4 \mathrm{~b}$ ). In addition, the ADR in the left colon was also higher than that in the PEG group with borderline statistical significance $(13.8 \%$ vs $10.0 \%, P=0.04)$.

The subgroup analysis revealed a significative increase of $A D R$ in the studies from Asian in the PEG with simethicone group $(26.8 \%$ vs. $20.7 \%, O R=1.45$, $95 \% \mathrm{Cl} 1.12-1.87, \mathrm{P}=0.005$ ) (Fig. $4 \mathrm{C}$ ), and the baseline $A D R<25 \%$ of the studies included was associated with the significant benefit of simethicone $(\mathrm{OR}=1.55,95 \% \mathrm{Cl} 1.16-2.07, \mathrm{P}=0.003)$ (Fig. $4 \mathrm{~d}$ ). In addition, our analysis revealed that there was no significant difference of ADR between the two groups with respect to the dose of simethicone, suggesting that the low-dose simethicone was as effective as the high-dose one with respect to the detection of colorectal benign tumours.

The comparison of PDR between the two groups showed no differences for proportion of PDR, dose of simethicone, size of polyps, and the populations, when simethicone was added.

\section{Conclusions}

Simethicone is an effective antifoaming agent during endoscopic procedures. The Gastroenterological Society of Australia consensus panel found that the current evidence supported the use of simethicone for improving visibility and likely facilitates adenoma detection at colonoscopy [33]. In addition, a recently study reported that a $10 \%$ increase in the detection rate of colorectal polyp when simethicone is added to the water pump during colonoscopy [34].

Although simethicone adding to PEG solution could improve bowel cleanness and mucosal visibility [35], out results found that simethicone did not the affect the total ADR and PDR. It may be related to the possible explanation that solid stool was unlikely to be cleaned, despite simethicone could improve the overall bowel cleanness. ADR has been recognized as the most indicator for quality of the colonoscopy. The current international guidelines have recommended the ADR should be $\geq 25 \%$ overall as the minimal requirement of the surveillance colonoscopy [36] In our subgroup analysis, we revealed a positive effect of simethicone with statistical significance in the low ADR group ( $<25 \%)$ and the studies from Asia. An interesting finding in our study was that the population of the low ADR group were all from Asian. This phenomenon might be related to the gene, diet and microbiota of Asian.

Furthermore, our subgroup analysis revealed that simethicone could significantly improve the small ADR ( $10 \mathrm{~mm})$ of the proximal colon. The main reason is that simethicone can improve the bowel preparation, especially in the right colon [37]. As bubbles are more commonly present in the proximal colon, especially the ascending colon, the bubble elimination could enhance the ability to detect smaller proximal adenomas. Previous study revealed that missed cancers of the proximal colon were more often found in the poor bowel preparation [38]. But simethicone did not affect the ADR in the left colon significantly, which may be associated with the small sample of studies included. Therefore, further large clinical trials are necessary to confirm our results.

The addition of 2-3 $\mathrm{mL}$ of $120 \mathrm{mg} / \mathrm{mL}$ simethicone to lavage fluid was recommend [33]. In the subgroup analysis, we compared the effect of the low dose simethicone ( $<400 \mathrm{mg}$ ) versus that of high dose simethicone ( $\geq 400 \mathrm{mg}$ ) for ADR and PDR. Our results also revealed that simethicone with high or low dose had no significant difference of ADR and PDR, suggesting that the low-dose simethicone was not inferior to the high-dose one in terms of ADR and PDR, which is similar to the study of Li et al [39]. Although residual simethicone in biopsy channels could promote biofilm formation [40], simethicone adding to PEG solution could decrease the infection risk of endoscope-transmission [31]. However, the optimal dose of SIM is yet to be ascertained [41]. Further research is required to determine the optimal dose of simethicone in clinical practice.

The strengths of our study were as follows. First, the subgroup analyses and sensitivity analyses were performed to explore the potential causes. To minimize potential bias, we performed sensitivity analyses to assess the impact on the heterogeneity by excluding one or more studies at a time and performed subgroup analyses according to the site and size of colorectal benign tumours, the population included, and the proportion of ADR. There was no significant heterogeneity found in the meta-analysis of ADR, except for the right-side ADR. When Valiante's study was excluded, it no longer showed heterogeneity of PDR. Second, our results of the subgroup analyses for ADR and PDR included the population included and the dose of simethicone

Page $10 / 16$ 
before colonoscopy. Thirdly, 20 studies were eligible for our meta-analysis. A large number of included studies allowed for firm conclusions and adequate subgroup analyses. Therefore, the results of our study are convincing.

There are several limitations to our meta-analysis. First, our meta-analysis was restricted to publications written in English, which might have produced a potential selection bias. Second, all included studies were single-blinded for outcome assessment; therefore, further double-blind RCTs should be conducted to confirm the positive effects of simethicone. Third, demographic and procedure data, such as race, diet, microbiota, and genes, may have been interesting to evaluate, but those data were not analyzed due to the limited condition. Fourth, although endoscopists were trained adequately, the effect of observer bias cannot be ignored.

In conclusion, we believe that simethicone might benefit to improve small ADR, especially in the proximal colon, for colonoscopy in Asian with low baseline ADR. The simethicone with low dose was not inferior to that with high dose with respect to the detection of colorectal benign tumours. Additional large clinical trials are necessary to validate our results and to evaluate the ideal dose of simethicone.

\section{Abbreviations}

\section{PEG}

Polyethylene glycol; ADR:Colorectal adenoma detection rate; PDR:Colorectal polyp detection rate; CRC:Colorectal cancer; OR:Odds ratio; MD:Mean difference; SDM:Standardized difference in mean; Cls:Confidence intervals; PRISMA:Preferred Reporting Items for Systematic Reviews and MetaAnalysis; S:PEG with simethicone group; NS:PEG group only; Sim:Simethicone; N:Total number of patients included; NR:Not report; BBPS:Boston Bowel Preparation Scale;

\section{Declarations}

\section{Ethics approval and consent to participate}

Not applicable.

\section{Consent for publication}

Not applicable.

\section{Availability of data and materials}

A dataset including the data analyzed may be available from the corresponding author on reasonable request.

\section{Competing interests}

The authors declare that they have no conflicts of interest.

\section{Funding}

This project was supported by the Natural Science Foundation of Wuhan (grant number: WX18Y04). The funders had no role in the study design, data collection, and data analysis, interpretation of data and writing of the manuscript.

\section{Authors' contributions}

$\mathrm{HZ}$ (Heng Z) designed this study and critically revised the manuscript; $\mathrm{HZ}$ (Hu Z) and JL were responsible for data acquisition and extraction; $\mathrm{HZ}$ (Hu Z) drafted the manuscript, analyzed the data, and interpreted the results; $\mathrm{HZ}$ ( $\mathrm{Hu} \mathrm{Z}$ ), LM, and JL were involved in editing the manuscript; all authors read and approved the final manuscript.

\section{Acknowledgements}

Not applicable.

\section{Author details}

${ }^{1}$ Department of Gastroenterology, the Central Hospital of Wuhan, Tongji Medical College, Huazhong University of Science and Technology, Wuhan 430014, Hubei Province, China

2 Department of Gastroenterology, the Eighth Hospital of Wuhan, Wuhan 430014, Hubei Province, China

\section{References}

1. Ng SC, Wong SH. Colorectal cancer screening in Asia. Br Med Bull. 2013;105:29-42. DOI:10.1093/bmb/lds040]. [PMID: 23299409. 
2. Shin A, Kim KZ, Jung KW, Park S, Won YJ, Kim J, Kim DY, Oh JH. Increasing trend of colorectal cancer incidence in Korea, 1999-2009. Cancer Res Treat 2012; 44(4): 219 - 26 [PMID:23341785 DOI:10.4143/crt.2012.44.4.219].

3. Meester RG, Doubeni CA, Lansdorp-Vogelaar I, Jensen CD, van der Meulen MP, Levin TR, Quinn VP, Schottinger JE, Zauber AG, Corley DA, van Ballegooijen M. Variation in Adenoma Detection Rate and the Lifetime Benefits and Cost of Colorectal Cancer Screening: A Microsimulation Model. JAMA. 2015;313(23):2349-58. DOI:10.1001/jama.2015.6251]. [PMID:26080339.

4. Corley DA, Jensen CD, Marks AR, Zhao WK, Lee JK, Doubeni CA, Zauber AG, de Boer J, Fireman BH, Schottinger JE, Quinn VP, Ghai NR, Levin TR, Quesenberry CP. Adenoma detection rate and risk of colorectal cancer and death. N Engl J Med 2014; 370 (14): 1298 - 306 [PMID:24693890 DOI:10.1056/NEJMoa1309086].

5. Wexner SD, Beck DE, Baron TH, Fanelli, Robert D, Hyman N, Shen B, Wasco KE. A consensus document on bowel preparation before colonoscopy: prepared by a task force from the American Society of Colon and Rectal Surgeons (ASCRS), the American Society for Gastrointestinal Endoscopy (ASGE), and the Society of American Gastrointestinal and Endoscopic Surgeons (SAGES). Gastrointest Endosc. 2006;63(7):894-909. DOI:10.1016/j.gie.2006.03.918]. [PMID:16733101.

6. Lebwohl B, Kastrinos F, Glick M, et al. The impact of suboptimal bowel preparation on adenoma miss rates and the factors associated with early repeat colonoscopy. Gastrointest Endosc. 2011;73:1207-14.

7. Clark BT, Protiva P, Nagar A, Imaeda A, Ciarleglio MM, Deng Y, et al. Quantification of Adequate Bowel Preparation for Screening or Surveillance Colonoscopy in Men. Gastroenterology. 2016;150(2):396-405; quiz e14-5. Epub 2015/10/07. doi:10.1053/j.gastro.2015.09.041. PubMed PMID: 26439436; PubMed Central PMCID: PMCPMC4728019.

8. Bugajski M, Wieszczy P, Hoff G, Rupinski M, Regula J, Kaminski MF. Modifiable factors associated with patient-reported pain during and after screening colonoscopy. Gut. 2018;67(11):1958-64. doi:10.1136/gutjnl-2017-313905. PubMed PMID: 28970289. Epub 2017/10/04.

9. Horton N, Garber A, Hasson H, Lopez R, Burke CA. Impact of Single-vs. Split-Dose Low-Volume Bowel Preparations on Bowel Movement Kinetics, Patient Inconvenience, and Polyp Detection: A Prospective Trial. Am J Gastroenterol. 2016;111(9):1330-7. doi:10.1038/ajg.2016.273. PubMed PMID: 27377521. Epub 2016/07/06.

10. Higgins JP, Altman DG, Gøtzsche PC, Jüni P, Moher D, Oxman AD, Savovic J, Schulz KF, Weeks L. The Cochrane Collaboration's tool for assessing risk of bias in randomised trials. BMJ. 2011;343:d5928. DOI:10.1136/bmj.d5928]. [PMID:22008217.

11. Higgins JP. Commentary. Heterogeneity in meta-analysis should be expected and appropriately quantified. Int J Epidemiol. 2008;37(5):1158-60. DOI:10.1093/ije/dyn204]. [PMID:18832388.

12. Moher D, Shamseer L, Clarke M, Ghersi D, Liberati A, Petticrew M, Shekelle P, Stewart LA. Preferred reporting items for systematic review and metaanalysis protocols (PRISMA-P) 2015 statement. Syst Rev. 2015;4:1. DOI:10.1186/2046-4053-4-1]. [PMID:25554246.

13. Zhang S, Zheng DP, Wang JP, Wu J, Lei P, Luo Q, Wang L, Zhang B, Wang H, Cui Y, Chen M. Simethicone improves bowel cleansing with low-volume polyethylene glycol: a multicenter randomized trial. Endoscopy. 2018;50(4):412-22. DOI:10.1055/s-0043-121337]. [PMID:29132175.

14. Bai Y, Fang J, Zhao SB, Wang D, Li YQ, Shi RH, Sun ZQ, Sun MJ, Ji F, Si JM, Li ZS. Impact of preprocedure simethicone on adenoma detection rate during colonoscopy: a multicenter endoscopist-blinded randomized controlled trial. Endoscopy. 2018;50(2):128-36. DOI:10.1055/s-0043-119213]. [PMID: 28985630.

15. Yoo IK, Jeen YT, Kang SH, Lee JH, Kim SH, Lee JM, Choi HS, Kim ES, Keum B, Chun HJ, Lee HS, Kim CD. Improving of bowel cleansing effect for polyethylene glycol with ascorbic acid using simethicone: A randomized controlled trial. Medicine(Baltimore) 2016; 95(28): e4163 [PMID:27428209 DOI:10.1097/MD.0000000000004163].

16. Zorzi M, Valiante F, Germanà B, Baldassarre G, Coria B, Rinaldi M, Heras Salvat H, Carta A, Bortoluzzi F, Cervellin E, Polo ML, Bulighin G, Azzurro M, Di Piramo D, Turrin A, Monica F. Comparison between different colon cleansing products for screening colonoscopy.A noninferiority trial in populationbased screening programs in Italy. Endoscopy 2016; 48(3): 223 - 31 [PMID:26760605 DOI:10.1055/s-0035-1569574].

17. Kump P, Hassan C, Spada C, Brownstone E, Datz C, Haefner M, Renner F, Schoefl R, Schreiber F. Efficacy and safety of a new low-volume PEG with citrate and simethicone bowel preparation for colonoscopy (Clensia): a multicenter randomized observer-blind clinical trial vs. a low-volume PEG with ascorbic acid (PEG-ASC). Endosc Int Open. 2018;6(8):E907-E. DOI:10.1055/a-0624-2266]. PMID:30083580.

18. Parente F, Vailati C, Bargiggia S, Manes G, Fontana P, Masci E, Arena M, Spinzi G, Baccarin A, Mazzoleni G, Testoni PA. 2-Litre polyethylene glycolcitrate- simethicone plus bisacodyl versus 4-litre polyethylene glycol as preparation for colonoscopy in chronic constipation. Dig Liver Dis 2015; 47(10): 857 - 63 [PMID:26232311 DOI:10.1016/j.dld.2015.06.008].

19. Mussetto A, Frazzoni L, Paggi S, Dari S, Laterza L, Radaelli F, Hassan C, Triossi O, Fuccio L. Split dosing with a low-volume preparation is not inferior to split dosing with a high-volume preparation for bowel cleansing in patients with a history of colorectal resection: a randomized trial. Endoscopy 2015; 47(10): 917 - 24 [PMID: 25910064 DOI:10.1055/s-0034-1391987].

20. de Leone A, Tamayo D, Fiori G, Ravizza D, Trovato C, De Roberto G, Fazzini L, Dal Fante M, Crosta C. Same-day 2-L PEG-citrate- simethicone plus bisacodyl vs split 4-L PEG: Bowel cleansing for late-morning colonoscopy. World J Gastrointest Endosc 2013; 5(9): 433-9 [PMID:24044042 DOI:10.4253/wjge.v5.i9.433].

21. 10.3748/wjg.v19.i33.5493]

Valiante F, Bellumat A, De Bona M, De Boni M. Bisacodyl plus split 2-L polyethylene glycol-citrate- simethicone improves quality of bowel preparation before screening colonoscopy. World J Gastroenterol 2013;19(33): p. 5493-9 [PMID: 24023492 DOI: 10.3748/wjg.v19.i33.5493].

22. 10.1016/j.dld.2012.07.011]

Page 12/16 
Cesaro P, Hassan C, Spada C, Petruzziello L, Vitale G, Costamagna G. A new low-volume isosmotic polyethylene glycol solution plus bisacodyl versus split-dose $4 \mathrm{~L}$ polyethylene glycol for bowel cleansing prior to colonoscopy: a randomised controlled trial. Dig Liver Dis 2013; 45(1): p. 23 - 7 [PMID: 22917636 DOI: 10.1016/j.dld.2012.07.011].

23. $10.1097 /$ SLE.0b013e31828e389d]

Gentile M, De Rosa M, Cestaro G, Forestieri P. 2 L PEG plus ascorbic acid versus 4 L PEG plus simethicone for colonoscopy preparation: a randomized single-blind clinical trial. Surg Laparosc Endosc Percutan Tech 2013; 23(3): p. 276 - 80 [PMID: 23751992 DOI:

10.1097/SLE.0b013e31828e389d].

24. $10.1038 / \operatorname{ctg} .2012 .16]$

Matro R, Tupchong K, Daskalakis C, Gordon V, Katz L, Kastenberg D. The effect on colon visualization during colonoscopy of the addition of simethicone to polyethylene glycol-electrolyte solution: a randomized single-blind study. Clin Trans/ Gastroentero/ 2012 ; 3 : p. e26 [PMID: 23238113 DOI: $10.1038 / \operatorname{ctg} .2012 .16]$.

25. Repici A, Cestari R, Annese V, Biscaglia G, Vitetta E, Minelli L, Trallori G, Orselli S, Andriulli A, Hassan C. Randomised clinical trial: low-volume bowel preparation for colonoscopy - a comparison between two different PEG-based formulations. Aliment Pharmacol Ther 2012; $36(8)$ : p. 717 - 24 [PMID: 22924336 DOI:10.1111/apt.12026].

26. Jansen SV, Goedhard JG, Winkens B, van Deursen CT. Preparation before colonoscopy: a randomized controlled trial comparing different regimes. Eur J Gastroenterol Hepatol. 2011;23(10):897-902. DOI:10.1097/MEG.0b013e32834a3444]. [PMID: 21900786.

27. 10.3748/wjg.v17.i42.4689]

Pontone S, Angelini R, Standoli M, Patrizi G, Culasso F, Pontone P, Redler A. Low-volume plus ascorbic acid vs high-volume plus simethicone bowel preparation before colonoscopy. World J Gastroenterol 2011; 17(42): p. 4689-95 [PMID:22180711 DOI:10.3748/wjg.v17.i42.4689].

28. Lazzaroni M, Petrillo M, Desideri S, Bianchi PG. Efficacy and tolerability of polyethylene glycol-electrolyte lavage solution with and without simethicone in the preparation of patients with inflammatory bowel disease for colonoscopy. Aliment Pharmacol Ther. 1993;7(6):655-9. DOI:10.1111/j.1365-2036.1993.tb00148.x]. [PMID:8161673.

29. McNally PR, Maydonovitch CL, Wong RK. The effect of simethicone on colonic visibility after night-prior colonic lavage. A double-blind randomized study. J Clin Gastroenterol. 1989;11(6):650-2. DOI:10.1097/00004836-198912000-00010]. [PMID:2584664.

30. McNally PR, Maydonovitch CL, Wong RK. The effectiveness of simethicone in improving visibility during colonoscopy: a double-blind randomized study. Gastrointest Endosc. 1988;34(3):255-8. DOI:10.1016/s0016-5107(88)71324-3]. [PMID:3292345.

31. Moraveji S, Casner N, Bashashati M, Garcia C, Dwivedi A, Zuckerman MJ, Carrion A, Ladd AM. The role of oral simethicone on the adenoma detection rate and other quality indicators of screening colonoscopy: a randomized, controlled, observer-blinded clinical trial. Gastrointest Endosc. 2019;90:141-9. DOI:10.1016/j.gie.2019.03.018]. [PMID:30926430.

32. Rishi M, Kaur J, Ulanja M, Manasewitsch N, Svendsen M, Abdalla A, Vemala S, Kewanyama J, Singh K, Singh N, Gullapalli N, Osgard E. Randomized, double-blinded, placebo-controlled trial evaluating simethicone pretreatment with bowel preparation during colonoscopy. World $\mathrm{J}$ Gastrointest Endosc. 2019;11(6):413-23. DOI:10.4253/wjge.v11.i6.413]. [PMID:31236194.

33. Devereaux BM, Taylor AC, Athan E, Wallis DJ, Brown RR, Greig SM, Bailey FK, Vickery K, Wardle E, Jones DM. Simethicone use during gastrointestinal endoscopy: Position statement of the Gastroenterological Society of Australia. J Gastroenterol Hepatol 2019; 1440-746 [PMID: 31242327 DOI:10.1111/jgh.14757].

34. Kutyla M, O'Connor S, Gurusamy AR, Gururatsakul M, Gould K, Whaley A, Kendall BJ, Hourigan L, Holtmann GJ. Influence of simethicone added to the rinse water during colonoscopies on polyp detection rates: Results of an unintended cohort study. Digestion. 2018;98:217-21. [PMID: 30045043 DOI: 10.1159/000489304].

35. Pan P, Zhao SB, Li BH, Meng QQ, Yao J, Wang D, et al. Effect of supplemental simethicone for bowel preparation on adenoma detection during colonoscopy: A meta-analysis of randomized controlled trials. J Gastroenterol Hepatol. 2019;34(2):314-20. doi:10.1111/jgh.14401. PubMed PMID: 30069899. Epub 2018/08/03.

36. Rex DK, Boland CR, Dominitz JA, Giardiello FM, Johnson DA, Kaltenbach T, Levin TR, Lieberman D, Robertson DJ. Colorectal Cancer Screening: Recommendations for Physicians and Patients from the U.S. Multi-Society Task Force on Colorectal Cancer. Gastroenterology. 2017;153(1):307-23. DOI:10.1053/j.gastro.2017.05.013]. [PMID: 28600072.

37. Benmassaoud A, Parent J. Canadian Association of Gastroenterology position statement on the impact of simethicone on endoscope reprocessing. J Can Assoc Gastroenterol. 2018;1(1):40-2. DOI:10.1093/jcag/gwx002]. [PMID: 31294395.

38. Doubeni CA, Corley DA, Quinn VP, Jensen CD, Zauber AG, Goodman M, et al. Effectiveness of screening colonoscopy in reducing the risk of death from right and left colon cancer: a large community-based study. Gut. 2018;67(2):291-8. doi:10.1136/gutjnl-2016-312712. PubMed PMID: 27733426; PubMed Central PMCID: PMCPMC5868294. Epub 2016/11/02.

39. Li DF, Luo MH, Du QQ, et al. Efficacy of Low-vs. High-Dose Simethicone with Polyethylene Glycol for Bowel Preparation: A Prospective Randomized Controlled Trial. J Gastroenterol Hepatol. 2020: 1440-746 DOI:10.1111/jgh.15022.

40. Barakat MT, Huang RJ, Banerjee S. Simethicone is retained in endoscopes despite reprocessing: impact of its use on working channel fluid retention and adenosine triphosphate bioluminescence values (with video). Gastrointest Endosc. 2019;89:115-23. DOI:10.1016/j.gie.2018.08.012]. [PMID: 30125574 
41. Hassan C, East J, Radaelli F, Spada C, Benamouzig R, Bisschops R, et al. Bowel preparation for colonoscopy: European Society of Gastrointestinal Endoscopy (ESGE) Guideline -Update 2019. Endoscopy. 2019;51(8):775-94. doi:10.1055/a-0959-0505. PubMed PMID: 31295746. Epub 2019/07/12.

Figures

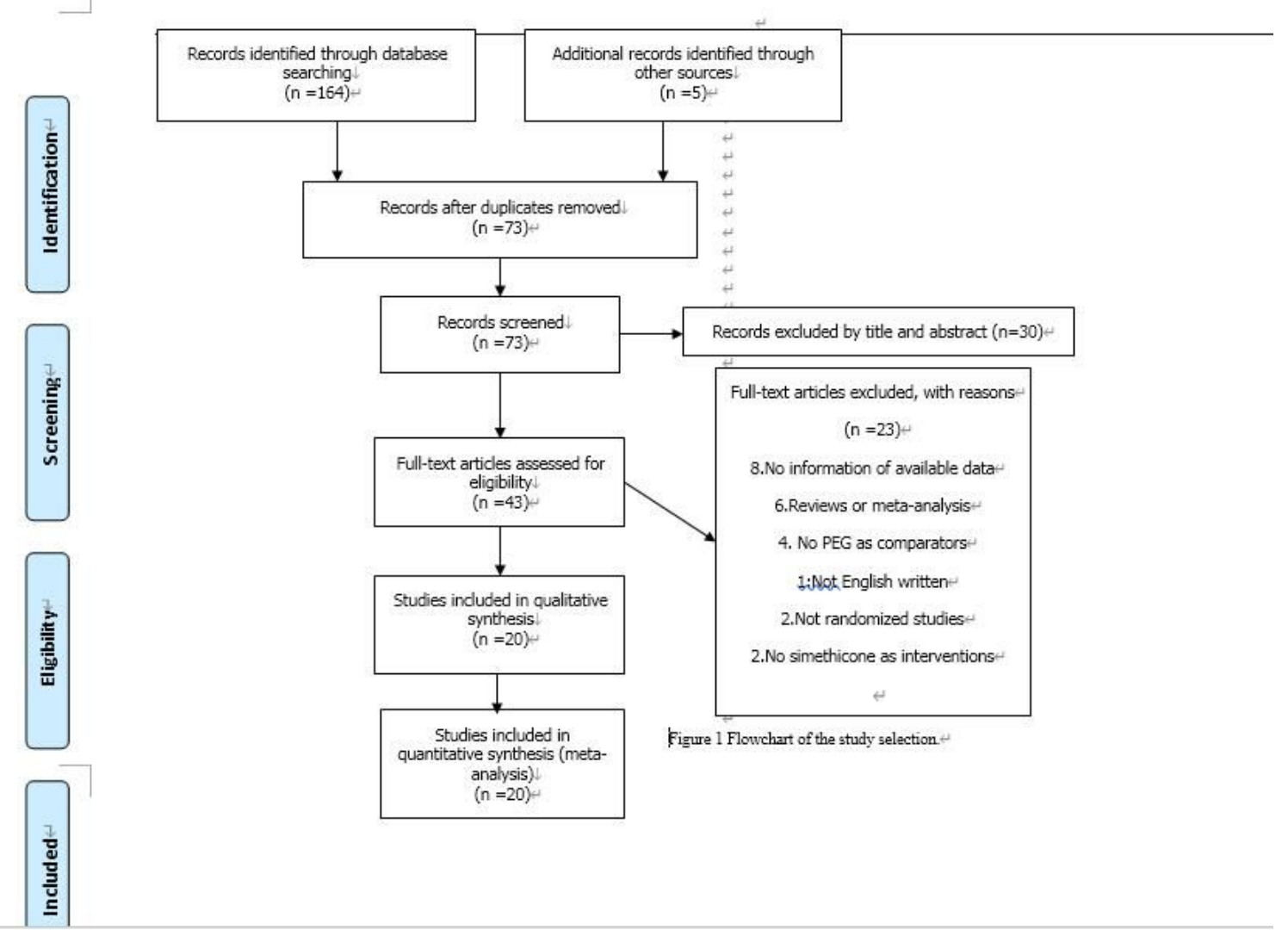

Figure 1

flowchart of the study selection

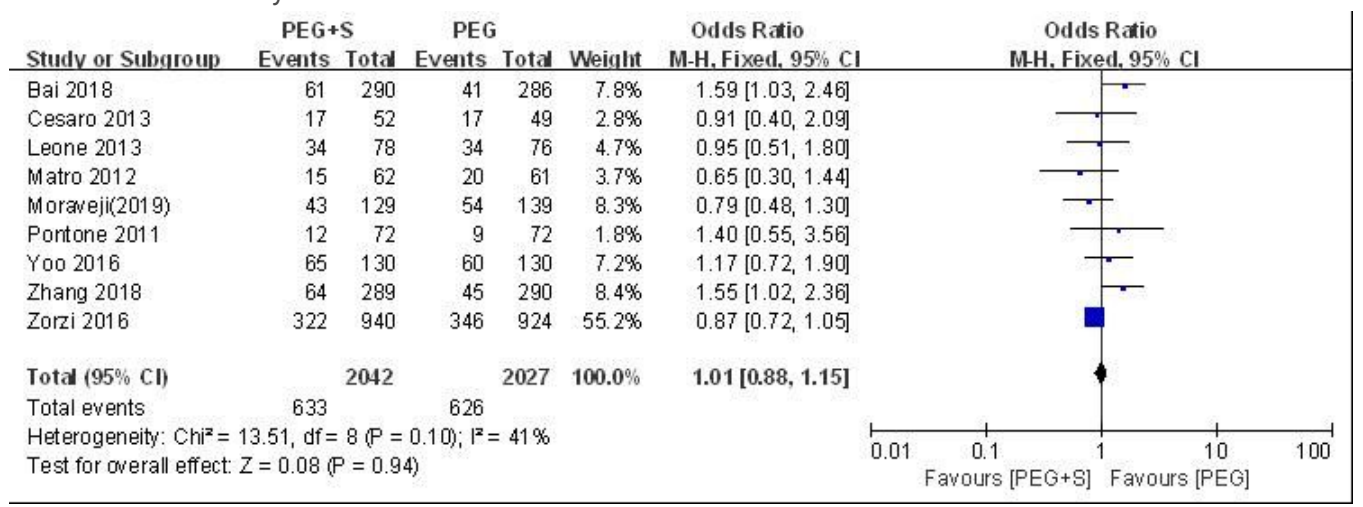

\section{Figure 2}

Fig 2 
PEG+S PEG Odds Ratio Odds Ratio

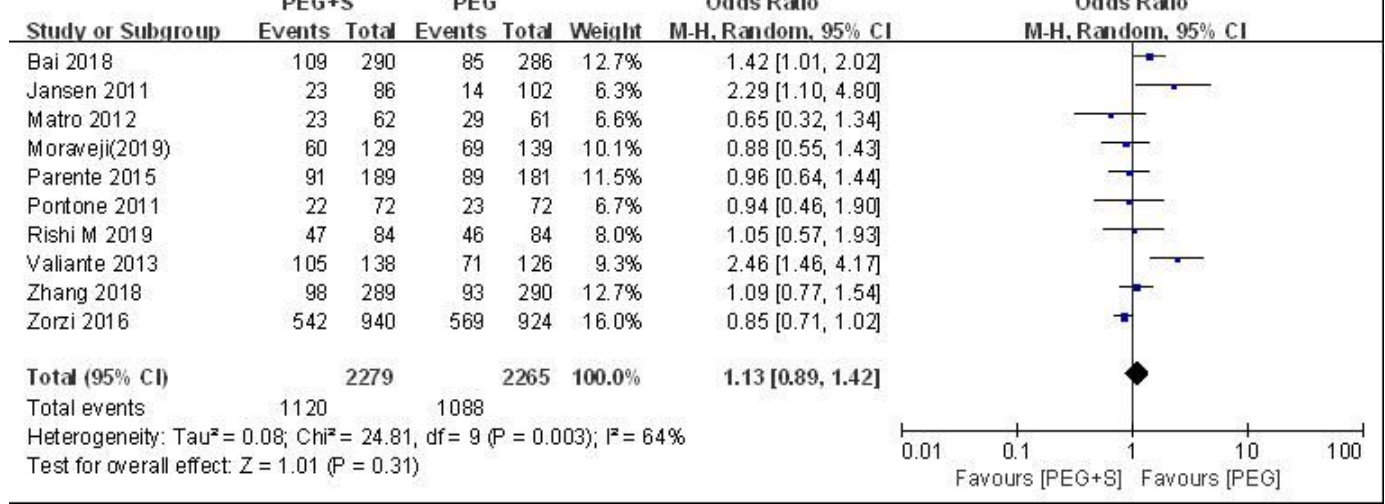

\section{Figure 3}

Fig 3

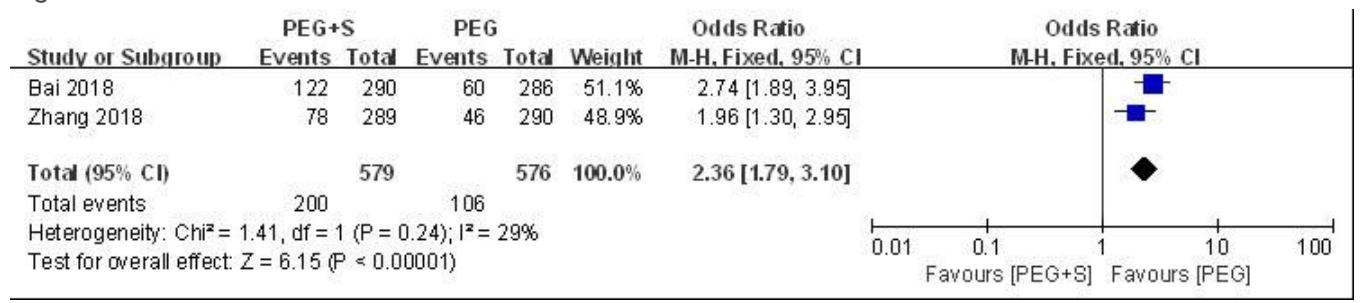

\section{Figure 4}

Fig 4

$$
\text { PEG+S PEG Odds Ratio }
$$

Odds Ratio

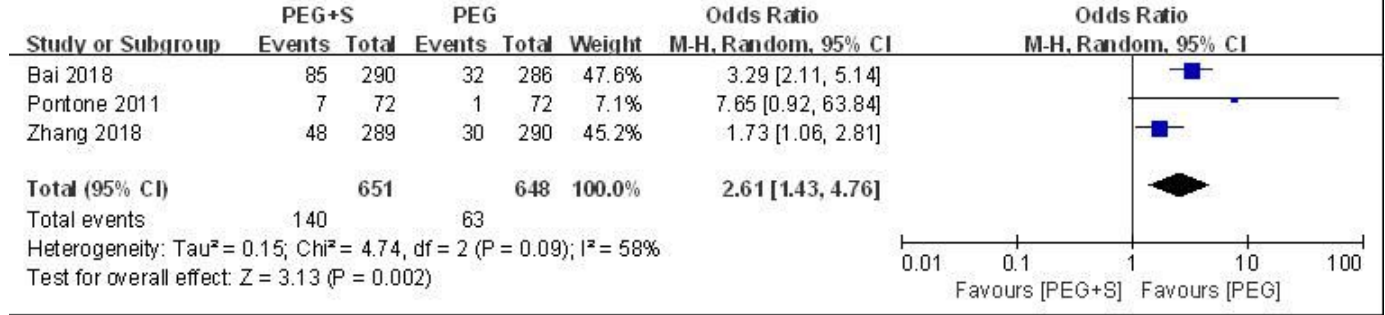

\section{Figure 5}

Fig 5

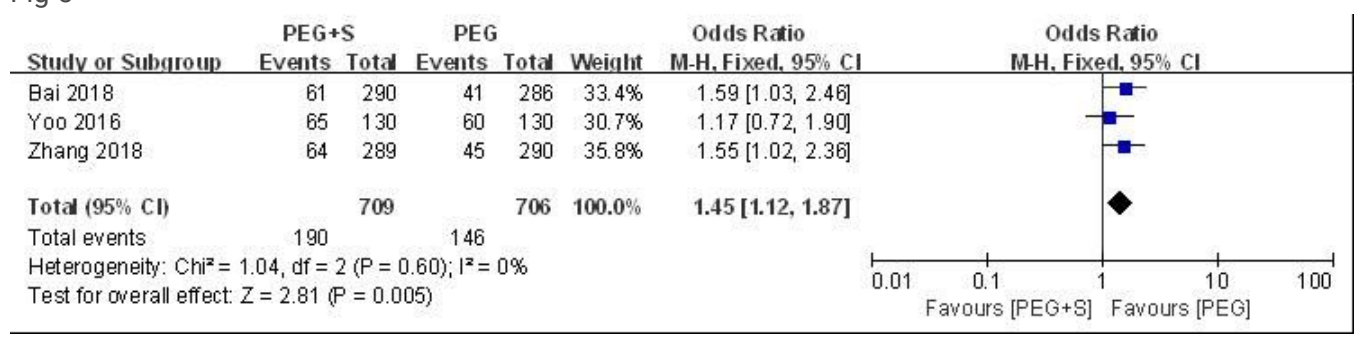

\section{Figure 6}

Fig 6

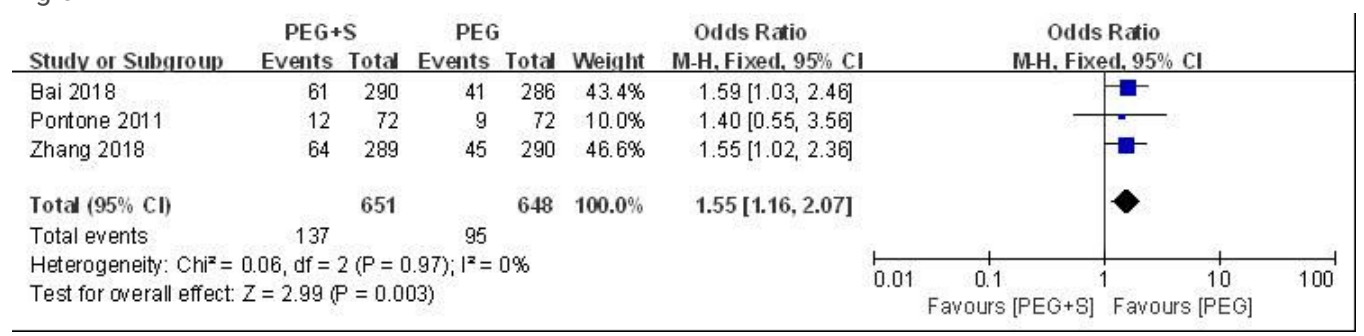


Fig 7

\section{Supplementary Files}

This is a list of supplementary files associated with this preprint. Click to download.

- SMSupplementarytable.docx

- PRISMA2009checklist.doc 